\title{
Eficácia das lactonas macrocíclicas sistêmicas (ivermectina e moxidectina) na terapia da demodicidose canina generalizada
}

\author{
[Efficacy of systemics macrocyclic lactones (ivermectin and moxidectin) for the treatment \\ of generalized canine demodicosis] \\ E.H. Delayte, M. Otsuka, C.E. Larsson, R.C.C. Castro \\ Faculdade de Medicina Veterinária e Zootecnia - USP \\ Av. Dr. Orlando Marques de Paiva \\ 8705509-000 - São Paulo, SP
}

\begin{abstract}
RESUMO
Avaliou-se a eficácia de lactonas macrocíclicas (ivermectina e moxidectina) sobre a eventual ocorrência de efeitos colaterais e acompanharam-se, após a alta parasitológica, por 12 meses, os cães tratados, visando detectar a recidiva do quadro dermatopático. Dos 63 animais, 59\% eram fêmeas, $76 \%$ apresentavam precisa definição racial e $67 \%$ tinham pelame curto. A ivermectina $(0,6 \mathrm{mg} / \mathrm{kg} / \mathrm{dia})$ foi administrada por via oral a 31 cães, e a moxidectina $(0,5 \mathrm{mg} / \mathrm{kg} / \mathrm{cada} 72$ horas $)$, pela mesma via, a 32 animais. Os tempos médios para a obtenção da primeira negativação do exame parasitológico do raspado cutâneo e para a consecução da alta foram, respectivamente, de 90 e 130 dias para a ivermectina e de 108 e 147 dias para a moxidectina. A ivermectina acarretou menos (16,1\%) efeitos colaterais em relação à moxidectina $(37,5 \%)(\mathrm{P}=0,03)$. As recidivas foram, respectivamente, $10,3 \%$ e $13 \%$ para ivermectina e moxidectina. Não houve diferença entre os dois protocolos de terapia quanto aos percentuais de recidiva $(\mathrm{P}=0,67)$ e eficácia $(\mathrm{P}=0,61)$. Ambas as lactonas macrocíclicas mostraram-se eficazes: ivermectina $89,7 \%$ e moxidectina $87 \%$.
\end{abstract}

Palavras-chave: cão, demodicidose, ivermectina, moxidectina, lactonas macrocíclicas

\begin{abstract}
The efficacy of ivermectin and moxidectin for treatment of generalized canine demodicosis, was evaluated to detect the eventual occurrence of side effects caused by the use of these drugs, and to follow the treated dogs for 12 months after obtaining parasitologic cure. Of 63 dogs, $59 \%$ were females, $76 \%$ were defined as purebred and $67 \%$ had short hair. Ivermectin $(0.6 \mathrm{mg} / \mathrm{kg} /$ daily) was orally administered to 31 dogs and moxidectin (0.5mg/kg/every 72 hours) to 32 dogs. The average number of days to obtain the first negative skin scraping results and the parasitologic cure were, respectively, 90 and 130 days for ivermectin, and 108 and 147 days for moxidectin. Ivermectin caused fewer side effects (16.1\%) than moxidectin (37.5\%) $(P<0.05)$. The percentages of relapse were, respectively, $10.3 \%$ and $13.0 \%$ when ivermectin and moxidectin were administered. No difference between protocols of therapy was found for percentage of relapse $(P \geq 0.67)$ and efficacy $(P \geq 0.61)$. Both drugs were effective and safe to treat generalized canine demodicosis: ivermectin $89.7 \%$ and moxidectin $87.0 \%$.
\end{abstract}

Keywords: canine demodicosis, demodectic mange, macrocyclic lactones, ivermectin, moxidectin

Recebido em 9 maio de 2003

Aceito em 11 de outubro de 2005

E-mail: adriano.delayte@sap.com 


\section{Delayte et al.}

\section{INTRODUÇ̃̃̃O}

A demodicidose canina (DC) é uma dermatose primária causada pela excessiva proliferação do Demodex canis - ácaro comensal da pele normaldecorrente de quadro herdado de imunodepressão mediada celularmente. No Hospital Veterinário da Faculdade de Medicina Veterinária e Zootecnia da Universidade de São Paulo, a DC representa $40 \%$ das ectoparasitoses atendidas.

A demodicidose pode ser classificada segundo sua distribuição corpórea (localizada ou generalizada) e faixa etária de ocorrência das primeiras manifestações (juvenil ou adulta) (Scott et al., 2001). A demodicidose localizada é, na maioria das vezes, auto-limitante, e a forma generalizada é considerada como uma das mais graves doenças cutâneas caninas (Paradis, 1999).

Muitas drogas têm sido usadas no tratamento da demodicidose canina. O amitraz, utilizado há mais de duas décadas, é a única droga aprovada para o tratamento da sarna demodécica pelo Food and Drug Administration (FDA). A eficácia desse clássico protocolo terapêutico apresenta grande variação, devido às diferentes formas de emprego. Na bibliografia consultada, podem-se observar percentis de eficácia variando entre 60 e 86\% (Mueller, 1983; Larsson e Gonçalves, 1984; Medleau e Willemse, 1995).

Como alternativas terapêuticas às situações de insucesso no tratamento com o amitraz, novas drogas têm sido preconizadas para a terapia de casos de DC generalizada, tais como ivermectina, milbemicina oxima, moxidectina e lufenuron (Paradis, 1999).

As avermectinas e as milbemicinas são lactonas macrocíclicas, classificadas como endectocidas. As primeiras incluem ivermectina, abamectina, doramectina e selamectina, sendo a própria milbemicina e a moxidectina duas milbemicinas. São produtos derivados da fermentação de actinomicetos do gênero Streptomyces, de ação anti-helmíntica e ectoparasiticida (Ayres e Almeida, 1999). Como ectoparasiticidas, essas substâncias são aprovadas no Brasil somente para uso em suínos, eqüinos e ruminantes.
Acredita-se que as lactonas macrocíclicas potencializem a ação inibidora neuronal mediada pelo ácido gama-aminobutírico (GABA), promovendo hiperpolarização do neurônio e, portanto, inibindo a transmissão nervosa (Sartor e Bicudo, 1999). Embora os mamíferos utilizem o GABA como neurotransmissor, as avermectinas e as milbemicinas geralmente não causam efeitos tóxicos neles, pois, por apresentarem alto peso molecular, não atravessam a barreira hematoencefálica e, portanto, não atuam no sistema nervoso central. No entanto, cães das raças Collie, Old English Sheepdog, Pastor de Shetland e Pastor Australiano, quando submetidos à terapia com ivermectina e milbemicina, podem manifestar sintomas de intoxicação, como convulsão, depressão, tremores, ataxia, letargia, emese, sialorréia e midríase, ou mesmo evoluir a óbito (Ayres e Almeida, 1999).

A ivermectina possui atividade contra o desenvolvimento de microfilárias de Dirofilaria immitis, sendo aprovada para a prevenção da dirofilariose canina, na dose de $6 \mu \mathrm{g} / \mathrm{kg} / \mathrm{mês}$ por via oral. No Brasil, está disponível comercialmente para a prevenção de dirofilariose, em especialidade farmacêutica de uso veterinário.

A moxidectina encontra-se disponível, em alguns países, como medicação preventiva de dirofilariose canina, na dose de $3 \mu \mathrm{g} / \mathrm{kg}$ (Paradis, 1999; Plumb, 1999; Wagner e Wendelberg, 2000). Como indicação extrabula da moxidectina, encontram-se dispostos na bibliografia médico-veterinária especializada (Bensignor e Carlotti, 1998; Pereira et al., 1999; Wagner e Wendelberg, 2000; Sousa et al., 2002) citações de seu emprego, pelas vias oral ou subcutânea, de solução injetável a $1 \%$ para a terapia da DC generalizada.

Os objetivos deste trabalho foram avaliar e comparar a eficácia da ivermectina e da moxidectina, administradas por via oral, no tratamento de cães com sarna demodécica generalizada, detectar a eventual ocorrência de efeitos colaterais e acompanhar, após a alta parasitológica, por 12 meses, os cães tratados, visando detectar a recidiva do quadro dermatopático. 


\section{MATERIAL E MÉTODOS}

Foram avaliados 63 cães com sarna demodécica generalizada (Tab. 1), diagnosticada por evidenciação de Demodex canis no exame parasitológico do raspado cutâneo (EPRC), 76\% eram de raça definida e $67 \%$ apresentavam pêlos curtos. Os animais foram, aleatoriamente, distribuídos em um dos dois protocolos empregados. No protocolo 1, incluíram-se 31 cães tratados com ivermectina, na apresentação de comprimidos elaborados especialmente por empresa farmacêutica veterinária para este estudo, na dose de $0,6 \mathrm{mg} / \mathrm{kg}$, por via oral, a cada 24 horas. No protocolo 2, agruparam-se 32 cães tratados com moxidectina, a partir de solução injetável a $1 \% 1$, na dose de $0,5 \mathrm{mg} / \mathrm{kg}$, a cada 72 horas, por via oral. Os animais foram submetidos aos protocolos até a obtenção da alta parasitológica. Nos casos de insucesso ou quando do eventual surgimento de efeitos colaterais, em que o proprietário optava pela suspensão da terapia, substituíam-se os protocolos em avaliação por aquele ortodoxo (amitraz em solução aquosa a 250ppm). Todos os cães foram submetidos à terapia tópica com xampu à base de peróxido de benzoíla a 2,5\%, a cada cinco dias. Os animais acometidos por piodermite foram tratados com cefalexina $(30 \mathrm{mg} / \mathrm{kg}$, via oral a cada 12 horas, por três a quatro semanas). Os retornos foram agendados a cada 30 dias, enquanto o EPRC se apresentava positivo e, a cada 15 dias, quando o EPRC tornou-se negativo. A alta parasitológica foi obtida após a obtenção de três EPRC negativos, com intervalo de 15 dias entre eles. Após a alta, os animais foram acompanhados por um ano, para a eventual constatação de recidiva.

Tabela 1. Distribuição segundo o sexo, faixa etária (meses) e peso corpóreo (quilogramas) dos cães com demodicidose generalizada submetidos ao tratamento com ivermectina ou moxidectina

\begin{tabular}{lccccc}
\hline & Macho & Fêmea & $\begin{array}{c}\text { Média de } \\
\text { idade (meses) }\end{array}$ & $\begin{array}{c}\text { Média de } \\
\text { peso (kg) }\end{array}$ & Total \\
\hline Ivermectina & 13 & 18 & 26,7 & 12,8 & 31 \\
Moxidectina & 13 & 19 & 19,2 & 21,3 & 32 \\
\hline
\end{tabular}

O teste de qui-quadrado foi aplicado para determinar a associação entre a presença de efeitos colaterais e o tipo de protocolo adotado, bem como entre a eficácia do tratamento e o tipo de protocolo (Bussab e Morettin, 2002). O teste exato de Fisher foi utilizado para comparar a ocorrência de recidiva entre os dois protocolos de terapia (Bussab e Morettin, 2002).

\section{RESULTADOS E DISCUSSÃO}

No tratamento com ivermectina, manteve-se a terapia até a obtenção da alta parasitológica em 29 cães $(93,6 \%)$. Nos dois (6,4\%) remanescentes, adotou-se o protocolo ortodoxo, após o surgimento de reações adversas consideradas graves pelos proprietários. Cinco animais $(16,1 \%)$ apresentaram reações adversas, porcentagem superior às relatadas por Ristic et al. (1995) e Gaguère (1996), respectivamente de $8,4 \%$ e $5,0 \%$. Os efeitos colaterais evidenciados foram ataxia $(3 / 31-9,7 \%)$, sonolência (3/31$9,7 \%)$, sialorréia $(2 / 31-6,5 \%)$, disorexia (1/31$3 \%$, prostração/apatia (1/31-3\%), alteração de comportamento/aumento da agressividade (1/31$3 \%)$.

A ataxia já havia sido observada quando da administração da ivermectina, diariamente, por via oral, para cães com DC generalizada, em dosagem similar de $600 \mu \mathrm{g} / \mathrm{kg}$ (Ristic et al., 1995; Gaguère, 1996) ou de $100 \mu \mathrm{g} / \mathrm{kg}$ (Mueller e Bettenay, 1999).

A porcentagem de manifestação de sialorréia foi próxima $(5 \%)$ à observada por Gaguère (1996). Esse autor usou a mesma posologia, via e

1 - Cydectin NF 1\% - Fort Dodge Saúde Animal Ltda 


\section{Delayte et al.}

freqüência de administração. A sialorréia foi também constatada em cães da raça Collie por Paul et al. (1987), ao avaliarem a toxicidade da ivermectina tanto na dosagem de $100 \mu \mathrm{g} / \mathrm{kg}$ quanto na de $600 \mu \mathrm{g} / \mathrm{kg}$.

Quanto à disorexia constatada em um único animal (3,2\%), apenas Lankas e Gordon (1989) descreveram quadro de anorexia em cão da raça Beagle, quando da administração de $2 \mathrm{mg} / \mathrm{kg}$ de ivermectina, dosagem três vezes superior à empregada neste estudo.

Um dos animais incluídos no protocolo 1 apresentou-se prostrado. Mueller e Bettenay (1999) relataram que dois cães com DC generalizada apresentaram-se relutantes a exercícios e letárgicos com doses diárias de 150 e $300 \mu \mathrm{g} / \mathrm{kg}$ de ivermectina. Um animal da raça Cocker Spaniel apresentou alteração de comportamento, caracterizada por aumento da agressividade. Paul et al. (1987) também observaram alterações do estado mental em cães da raça Collie, quando da administração de 100 e $2500 \mu \mathrm{g} / \mathrm{kg}$ de ivermectina.

Vários autores observaram, com freqüência, midríase em cães com DC generalizada submetidos à terapia com a ivermectina (Ristic et al., 1995; Gaguère, 1996; Mueller e Bettenay, 1999). Neste estudo, nenhum cão incluído no protocolo 1 apresentou tal alteração de diâmetro pupilar.

Em um dos dois animais submetidos ao protocolo ortodoxo, observaram-se disorexia, sonolência e ataxia após uma semana de terapia e, no outro, um quadro de ataxia. Após a suspensão da administração da ivermectina, houve total remissão dos sintomas em ambos os animais.

O tempo médio de terapia necessário para a primeira negativação do EPRC foi de $90 \pm 8$ dias (Fig. 1), inferior aos 147,3 dias observado por Gaguère (1996) e superior ao relatado por Ristic et al. (1995), de 45,5 dias. O tempo médio de terapia para a obtenção da alta parasitológica foi de $130 \pm 7$ dias, ultrapassando aquela obtida por Ristic et al. (1995), de 70 dias (Fig. 2 e 3).

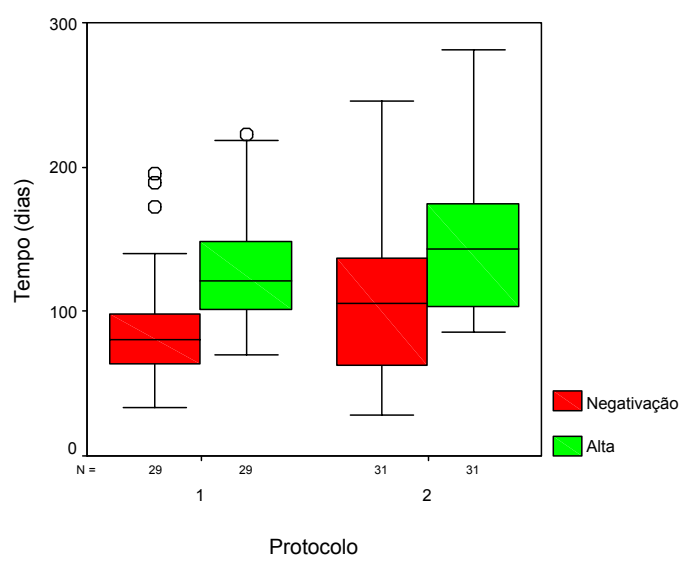

Figura 1. Tempo médio de terapia (dias) necessário para obtenção do primeiro exame do raspado cutâneo negativo e para alta parasitológica nos cães submetidos à terapia com o protocolo 1 (ivermectina - $0,6 \mathrm{mg} / \mathrm{kg} / 24 \mathrm{~h}$ ) ou com o protocolo 2 (moxidectina $-0,5 \mathrm{mg} / \mathrm{kg} / 72 \mathrm{~h}$ ).

Três cães apresentaram recidiva antes de completar um ano, a contar da alta. Com relação à ocorrência de $10,3 \%$ de recidivas nos animais submetidos ao protocolo 1 , resultado inferior aos percentuais de $16,7 \%$ e de $25 \%$ relatados, respectivamente, por Ristic et al. (1995) e Gaguère (1996), que administraram a lactona no mesmo esquema posológico deste estudo. O recrudescimento da DC generalizada já havia sido observado por outros autores (Medleau et al., 1995, citado por Fondati, 1996), pois 15 a $40 \%$ dos cães portadores da doença tiveram recidivas quando do emprego de, respectivamente, $600 \mu \mathrm{g} / \mathrm{kg}$ ou $400 \mu \mathrm{g} / \mathrm{kg}$ de ivermectina, diariamente.

Dos 32 cães que receberam a moxidectina, 31 $(96,8 \%)$ obtiveram alta parasitológica. Em um animal adotou-se o protocolo ortodoxo com o amitraz. Desses, $12 \quad(37,5 \%)$ apresentaram reações adversas. As manifestações adversas evidenciadas foram emese $(8 / 32-25 \%)$, disorexia $(2 / 32-6,3 \%)$, anorexia plena $(2 / 32-6,3 \%)$, sialorréia $(2 / 32-6,3 \%)$, adipsia $(1 / 32-3 \%)$, diarréia (1/32-3\%), sonolência (4/32-12,5\%), prostração/apatia $(2 / 32-6,3 \%)$, mioclonias $(1 / 32-$ $3 \%$ ) e enantema $(1 / 32-3 \%)$. 
Apesar da alta ocorrência (37,5\%) de reações adversas, apenas em um animal, que apresentou anorexia, adipsia e prostração já na manhã seguinte à terapia, optou-se pela adoção do protocolo ortodoxo. Este valor percentual de ocorrência de efeitos colaterais $(37,5 \%)$ foi superior àqueles relatados por Burrows (1997), Bensignor e Carlotti (1998), Wagner e Wendlberger (2000), Delayte et al. (2001) e Sousa et al. (2002), respectivamente, 11\%, 5,5\%, $14 \%, 25,0 \%$ e $5,9 \%$, quando da administração da moxidectina por via oral nas doses de 0,3 a $1,0 \mathrm{mg} / \mathrm{kg}$.

Os efeitos colaterais mais observados pelos proprietários dos animais submetidos ao protocolo 2 foram vômito (25\%) e sonolência (12,5\%). A ocorrência de vômitos já havia sido relatada, no Brasil, por Delayte et al. (2001), quando da administração de $0,5 \mathrm{mg} / \mathrm{kg} / 24$ horas, por via oral, e por Wagner e Wendlberger (2000), em apenas um cão (4,5\%). Deve-se, no entanto, destacar que a dosagem empregada foi de $0,4 \mathrm{mg} / \mathrm{kg}$ por via oral diariamente, ou seja, $20 \%$ inferior à utilizada neste trabalho. A letargia descrita por Bensignor e Carlotti (1998) em um cão $(5,5 \%)$ e por Wagner e Wendlberger (2000) (4,5\%), quando do emprego diário de $400 \mu / \mathrm{kg}$ de moxidectina por via oral, foi também relatada por Delayte et al. (2001), com doses de 0,5$1,0 \mathrm{mg} / \mathrm{kg}$, a cada 72 horas, tanto por via oral quanto por via subcutânea.

As manifestações de anorexia observadas por vários autores, quando administraram a moxidectina em doses inferiores ao protocolo 2, foram de 11\% (Burrows, 1997), 5,5\% (Bensignor e Carlotti, 1998) e 4,5\% (Wagner e Wendlberger, 2000). Neste trabalho, a anorexia foi relatada pelos proprietários de dois dos cães $(6,2 \%)$ neste trabalho. Bensignor e Carlotti (1998) e Wagner e Wendlberger (2000) evidenciaram, também, como efeito adverso, a ataxia, sintoma não observado neste trabalho.

A ocorrência de mioclonias em 3\% dos animais submetidos ao protocolo 2 foi também observada por Sousa et al. (2002) em 5,9\% dos cães, quando administraram moxidectina na dose de $1,0 \mathrm{mg} / \mathrm{kg}$ por via oral a cada 72 horas. A mioclonia não foi evidenciada quando se reduziu a dose para $0,5 \mathrm{mg} / \mathrm{kg}$.

Como houve alta ocorrência de vômitos (25\%) nos animais que receberam moxidectina, a eles administrou-se ranitidina na dosagem de $2 \mathrm{mg} / \mathrm{kg}$, que resultou em redução e até mesmo no desaparecimento de emese.

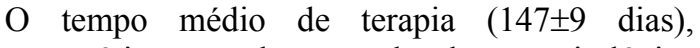
necessário para obtenção da alta parasitológica (Fig. 1, 4 e 5), foi semelhante aos obtidos por Bensignor e Carlotti (1998) e por Delayte et al. (2001), respectivamente, de 150 e 154 dias, e superou os 107 e 72 dias de terapia referidos por Burrows (1997) e Wagner e Wendlberger (2000).

Evidenciou-se recidiva da demodiciose em quatro animais (13\%) antes que se completasse um ano da alta. Considerando os resultados de 31 cães, com quatro (13\%) casos de recidiva dentre aqueles mantidos sob terapia com o protocolo 2 , verificou-se que o percentual de eficácia foi de $87 \%$.

O percentual de eficácia é inferior aos relatados por Pereira et al. (1999) e Wagner e Wendlberger (2000). Em ambos os trabalhos, a eficácia foi de $100 \%$, embora no primeiro a moxidectina tenha sido empregada por via subcutânea na dose de 0,5 a $1,0 \mathrm{mg} / \mathrm{kg}$, e no segundo, por via oral, na dose de 0,4mg $/ \mathrm{kg}$, diariamente. Burrows (1997) e Bensignor e Carlotti (1998), ao utilizarem a moxidectina nas dosagens, respectivamente, de $0,3 \mathrm{mg} / \mathrm{kg}$ e $0,4 \mathrm{mg} / \mathrm{kg}$ diariamente, por via oral, também relataram maior eficácia do tratamento $(89 \%)$.

O percentual de reações adversas à moxidectina, $37,5 \%$, foi superior $(\mathrm{P}<0,03)$ ao percentual, $16,1 \%$, observado nos casos tratados com ivermectina (Fig. 6). 


\section{Delayte et al.}

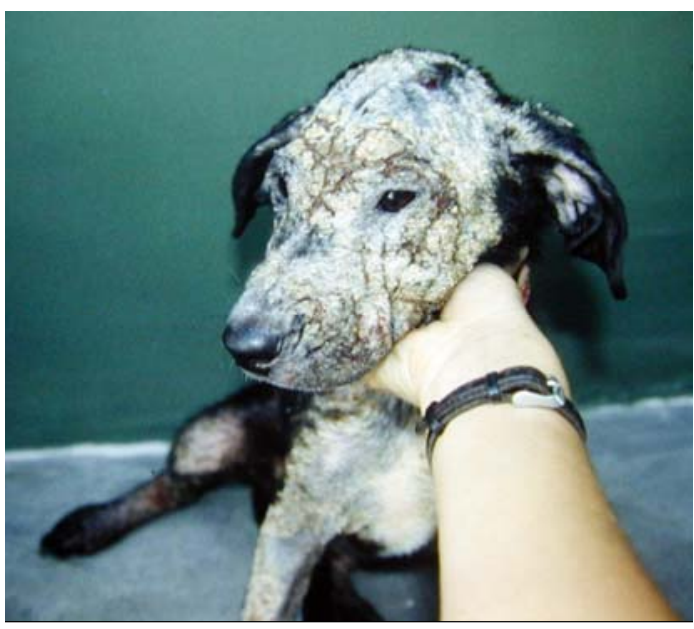

Figura 2. Cão, SRD, fêmea, seis meses, demodicidose canina generalizada, alopecia, crostas melicéricas em região cefálica, préterapia.

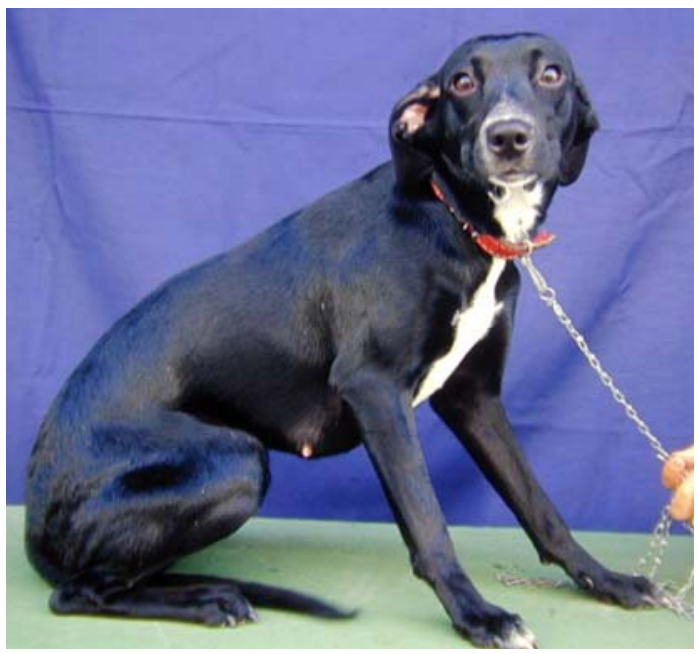

Figura 3. Remissão das lesões quando da alta parasitológica, após 155 dias de terapia com a ivermectina $(0,6 \mathrm{mg} / \mathrm{kg} / \mathrm{dia}$, via oral $)$.

Não se observou diferença quanto à recidiva entre ambos os protocolos $(\mathrm{P}=0,67)$ nem tampouco quanto às suas eficácias $(\mathrm{P}=0,61)$ nos casos de DC generalizada. Ambas as lactonas macrocíclicas mostraram-se eficazes, com percentuais muito próximos, ivermectina $89,7 \%$ e moxidectina $87,0 \%$.

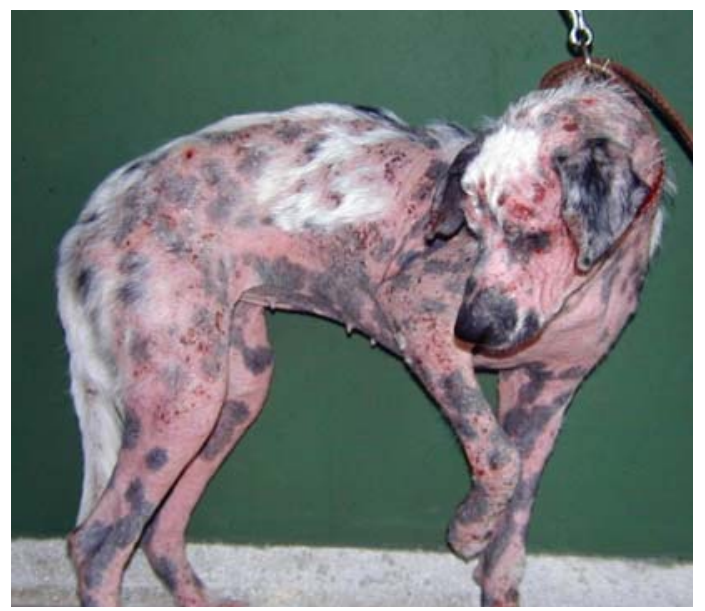

Figura 4. Cão, Dálmata, fêmea, 13 meses, demodicidose generalizada, falacrose, eritema em lençol, crostas hemorrágicas, préterapia.

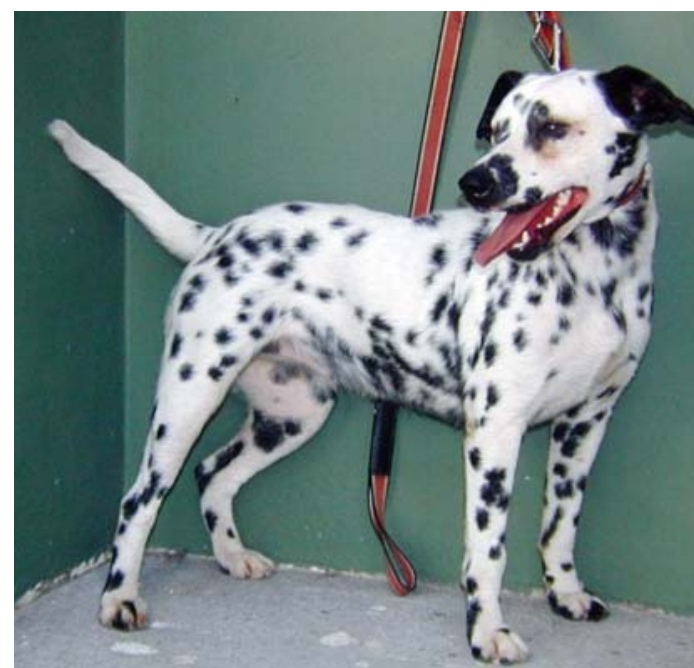

Figura 5. Remissão das lesões quando da alta parasitológica, após 199 dias de terapia com a moxidectina $(0,5 \mathrm{mg} / \mathrm{kg} / \mathrm{cada} 72$ horas, via oral).

A formulação de ivermectina, preparada sob a forma de comprimidos elaborados especialmente para este trabalho, a partir de produto de grau farmacêutico, não havia sido anteriormente utilizada e referida em outros trabalhos. Habitualmente, utiliza-se o princípio ativo contido em formulações (soluções injetável, oral ou tópica dosificada), destinadas ao tratamento e ao controle de enfermidades parasitárias dos animais de exploração zootécnica. 


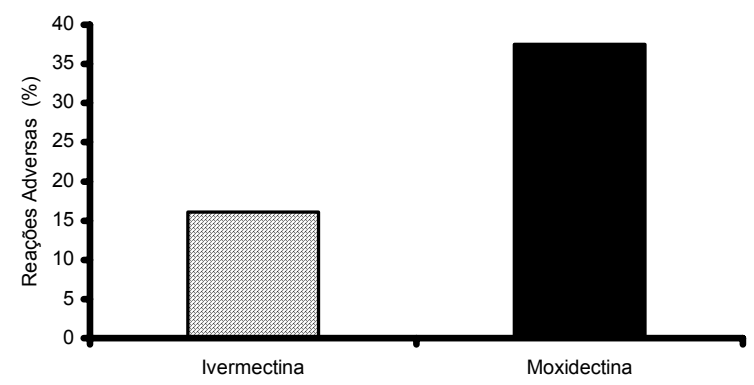

Figura 6. Freqüência (\%) de reações adversas observadas em cães com demodiciose submetidos à terapia com ivermectina $(0,6 \mathrm{mg} / \mathrm{kg} / 24 \mathrm{~h})$ e com moxidectina $(0,5 \mathrm{mg} / \mathrm{kg} / 72 \mathrm{~h})$.

Com relação à exeqüibilidade dos dois protocolos, ambos foram eficazes, de fácil administração, práticos, pouco onerosos e seguros para os cães tratados, sendo ambas as lactonas macrocíclicas, pelos resultados evidenciados, alternativas viáveis na terapia da DC generalizada.

Finalmente, o sucesso de qualquer tratamento da DC generalizada, além de envolver uma resposta à terapia pelo paciente, implica a disposição do proprietário em realizar o protocolo e comparecer aos retornos até a obtenção da alta e o compromisso de não utilizar o animal em cruzamentos, para evitar a propagação da doença, que pode ser perfeitamente controlada, senão totalmente curada.

\section{REFERÊNCIAS BIBLIOGRÁFICAS}

AYRES, M.C.C.; ALMEIDA, M.A. Agentes Antinematódeos. In: SPINOSA, H.S.; GÓRNIAK, S.L.; BERNARDI, M.M. Farmacologia aplicada à medicina veterinária. 2.ed. Rio de Janeiro: Guanabara Koogan, 1999. p.453-465.

BENSIGNOR E.; CARLOTTI, D.N. Essai de traitement de la démodécie généralisée du chien par la moxidectine: Résultats préliminaires (18 cas) [abstract 1]. In: GROUPE D'ETUDE DE DERMATOLOGIE VETERINAIRE (GEDAC); GROUPE DE TRAVAIL VETERINAIRES
BELGES (GTVB), 1998, Lille, France. Proceedings... [s.1.]: GEDAC, GTVB, 1998. p.13.

BURROWS, M. Evaluation of the clinical efficacy of moxidectin in the treatment of generalised demodicosis in the dog. Murdoch University Veterinary Hospital. Western Australia with a grant from Cynamid Websters. Fort Dodge Animal Health, 1997.

BUSSAB, W.O.; MORETTIN, P.A. Estatística básica. 5.ed. São Paulo: Saraiva, 2002. p.526.

DELAYTE, E.H.; LARSSON, C.E.; OTSUKA, M. et al. Terapia da demodiciose com moxidectina - efeitos colaterais e eficácia. In: CONGRESSO PAULISTA DE CLÍNICOS VETERINÁRIOS DE PEQUENOS ANIMAIS, 1., 2001, Águas de Lindóia, SP. Anais... Águas de Lindóia: [s.n.], 2001. p.19.

FONDATI, A. Efficacy of daily oral ivermectin in the treatment of 10 cases of generalized demodicosis in adult dogs. Vet. Dermatol., v.7, p.99-104, 1996.

GUAGUÈRE, E. Traitement de la démodécie généralisée du chien par l'ivermectine: à propos de 20 cas. Prat. Méd. Chirurg. Anim. Compag., v.31, p.33-40, 1996.

LANKAS, G.R.; GORDON, L.R. Toxicology. In: CAMPBELL, W.C. Ivermectin and abamectin. New York: Springer-Verlag, 1989. p.89-112.

LARSSON, C.E.; GONÇALVES, M.A. Aspectos clínico-laboratoriais da terapia da demodiciose canina generalizada com diamidina (amitraz): resultados parciais. In: CONGRESSO BRASILEIRO DE CLÍNICOS VETERINÁRIOS DE PEQUENOS ANIMAIS, 7., 1984, Rio de Janeiro. Anais... Rio de Janeiro, 1984.

MEDLEAU, L.; WILLEMSE, T. Efficacy of daily amitraz therapy for refractory, generalized demodicosis in dogs: two independent studies. $J$. Am. Anim. Hosp. Assoc., v.31, p.246-249, 1995.

MUELLER, G.H. Amitraz treatment of demodicosis. J. Am. Anim. Hosp. Assoc., v.19, p.1426-1429, 1983.

MUELLER, R.S.; BETTENAY, S.V. A proposed new therapeutic protocol for the treatment of canine mange with ivermectin. $J$. Am. Anim. Hosp. Assoc., v.35, p.77-80, 1999. 


\section{Delayte et al.}

PARADIS, M. New approaches to the treatment of canine demodicosis. Vet. Clin. North Am.: Small Anim. Pract., v.29, p.1425-1436, 1999.

PAUL, A.J.; TRANQUILI W.J.; SEWARD, R.L. et al. Clinical observations in Collies given ivermectin orally. Am. J. Vet. Res., v.48, p.684$685,1987$.

PEREIRA, E.C.P.; SONODA, M.C.; SUGUIEDA, S.M. et al. Uso subcutâneo de moxidectina no tratamento da demodiciose canina generalizada. In: CONGRESSO BRASILEIRO DE CLÍNICOS VETERINÁRIOS DE PEQUENOS ANIMAIS, 20., 1999, Águas de Lindóia. Anais... Águas de Lindóia: Anclivepa, 1999. p.10-11.

PLUMB, D.C. Veterinary drug handbook. 3.ed. Ames: Iowa State University, 1999. p.443-445.

RISTIC, Z.; MEDLEAU, L.; PARADIS, M. et al. Ivermectin for treatment of generalized demodicosis in dogs. J. Am. Vet. Med. Assoc., v.207, p.1308-1310, 1995.

SARTOR, I.F.; BICUDO, P.L. Agentes empregados no controle de ectoparasitas. In:
SPINOSA, H.S.; GÓRNIAK, S.L.; BERNARDI, M.M. Farmacologia aplicada à medicina veterinária. 2.ed. Rio de Janeiro: Guanabara Koogan, 1999. p.480-492.

SCOTT, D.W.; MULLER, W.H.; GRIFFIN, C.E. Small animal dermatology. 6.ed. Philadelphia: W.B. Saunders, 2001. p.423-516.

SOUSA, M.G.; GERARDI, D.G.; HIGA, A.C. et al. Retrospective study of the use of moxidectin in the treatment of canine demodicosis at the veterinary hospital of São Paulo State University - UNESP - Jaboticabal campus - Brasil. In: WORLD SMALL ANIMAL VETERINARY ASSOCIATION, 27.; FECAVA, 8.; CONGRESSO NACIONAL DA AVEPA, 37., 2002, Granada, Spain. Free communications book... Granada, 2002. p.173.

WAGNER, R.; WENDLBERGER, U. Field efficacy of moxidectin in dogs and rabbits naturally infested with Sarcoptes spp., Demodex spp. and Psoroptes spp. mites. Vet. Parasitol., v.93, p.149-158, 2000. 